\title{
Analyzing the interactions of circRNAs, miRNAs, and mRNAs to predict ceRNA networks in human acute type $A$ aortic dissection
}

maoxun huang

Jilin university

hulin piao

Jilin university

yong wang

Jilin university

weitie wang

Jilin university

bo li

Jilin university

zhicheng zhu

Jilin university

rihao xu

Jilin university

dan li

Jilin university

tiance wang

Jilin university

kexiang liu ( $\sim$ kxliu64@hotmail.com)

Jilin university, changchun, Jilin

Research article

Keywords: Aortic dissection; circRNAs; miRNAs; mRNAs; ceRNA

Posted Date: January 29th, 2020

DOI: https://doi.org/10.21203/rs.2.10144/v2

License: (c) (7) This work is licensed under a Creative Commons Attribution 4.0 International License.

Read Full License 
The authors have withdrawn this preprint from Research Square 